\title{
Les prix de traduction fabriquent-ils une image régulatrice du traducteur?
}

Juan Zapata, Université de Liège

Valérie Le Plouhinec, ATLF

Les prix de traduction s'avèrent déterminants dans l'analyse des mécanismes de consécration institutionnels et des discours tenus par les traducteurs sur leur métier et sur leurs pairs. Détenteurs de légitimité et espaces de production de la valeur, les prix participent à la fois aux luttes pour la détention d'un capital de consécration et à la construction d'une image publique $\mathrm{du}$ traducteur. Or, à la différence de leurs voisins littéraires, largement médiatisés et devenus un objet d'étude privilégié de la sociologie des champs, ${ }^{1}$ les prix de traduction n'ont pas encore attiré l'attention des chercheurs. La quasi-absence de publication des discours prononcés lors de la remise de prix, le plus souvent produits de façon improvisée, l'omission dans leurs pages institutionnelles des critères de composition du jury et des modes de sélection et de vote, ainsi que le peu d'intérêt qu'ils suscitent dans la presse, marquée par l'absence notoire d'entretiens avec les agents impliqués (lauréats et jurés), contribuent aussi à la méconnaissance de leurs mécanismes de fonctionnement et entravent le travail du chercheur.

Ce sont donc ces conditions de travail qui ont déterminé notre enquête et nous ont permis d'émettre quelques hypothèses. Tout d'abord, il s'agit d'éclairer la façon dont les prix de traduction construisent leur autorité, leur droit à la parole. Ces prix n'étant pas soumis aux logiques commerciales, leur légitimité repose uniquement sur le jugement des pairs. Cette caractéristique les distingue des prix littéraires, qui favorisent la confusion entre instances de diffusion et instances de légitimation (Ducas), et les écarte de tout soupçon de connivence entre l'agent consacrant et les pressions économiques externes. Ensuite, il s'agit de démontrer comment les prix de traduction fabriquent une image régulatrice du traducteur. En primant un seul secteur du champ de la traduction (la traduction d'ouvrages littéraires, majoritairement romanesques), ${ }^{2}$ et en privilégiant les langues perçues comme « difficiles » ainsi que les petites maisons d'édition qui portent leur attention sur les auteurs qui ne font pas partie d'un circuit de large diffusion, « l'univers des traducteurs reproduit la structure dualiste des champs de production culturelle » (Sapiro et Heilbron 3-4). Ce faisant, ils cherchent à imposer une image légitime du traducteur (dominant symboliquement et dominé économiquement) (Bourdieu 233-88) à laquelle sont associées certaines valeurs (rareté du produit, désintéressement, vocation,

\footnotetext{
${ }^{1}$ Voir Nathalie Heinich, L'épreuve de la grandeur. Prix littéraires et reconnaissance. Paris: La Découverte, 1999 ; Gisèle Sapiro. La Guerre des écrivains. Paris: Fayard, 1999 ; Sylvie Ducas. La littérature, à quel(s) prix ? Histoire des prix littéraires. Paris: La Découverte, 2013 ; Jérôme Meizoz. " La fabrique du fétiche. Sociologie des prix littéraires. " L'œil sociologique et la littérature. Genève: Slatkine, 2004.

${ }^{2}$ Notre étude ne portera pas sur les traducteurs techniques et interprètes. La majorité des prix de traduction en France sont consacrés uniquement aux traducteurs littéraires. Il existe également les prix de traduction audiovisuelle de l'ATAA, Association des traducteurs/adaptateurs de l'audiovisuel.
} 
création, singularité, etc.) (Heinich Les traducteurs) qui s'opposent aux valeurs propres de la logique marchande.

$\mathrm{Ne}$ disposant pas des discours prononcés lors de la remise des prix, à l'exception de quelques-uns qui nous ont été fournis par leurs auteurs ou par les organisateurs des prix, ou que nous avons nous-mêmes enregistrés, notre enquête repose sur un questionnaire adressé aux lauréats et aux jurés des quatre dernières années. Les prix sélectionnés (Grand prix de la ville d'Arles, Prix Laure Bataillon, Grand prix de traduction SGDL [Société des gens de lettres], Prix Baudelaire, Prix Gérard de Nerval, Prix Maurice-Edgar Coindreau et Prix Pierre-François Caillé) ont été étudiés selon un même protocole d'analyse cherchant à établir leur profil et leur position dans le champ français de la traduction. ${ }^{3}$ Cela nous a permis d'identifier leurs critères d'attribution (" explicites » et « implicites »), le mode de composition et de vote des jurys, la présélection des ouvrages, les tendances en matière de traducteurs primés, de langues sources et de maisons d'édition récompensées. ${ }^{4}$ En outre, cette grille d'analyse nous a permis de comparer les prises de position des lauréats et des jurés à avec leurs profils et le profil du prix auquel ils appartiennent. Enfin, nous avons organisé nos résultats selon deux grands axes: d'un côté, les instances de consécration et, de l'autre, les traducteurs récompensés.

\section{Mise en perspective du corpus analysé}

Nous devons tout d'abord signaler que, pour les raisons déjà évoquées, notre corpus se limite aux quatre dernières années (voir le tableau annexe $n^{0} 1$ ). Cette délimitation temporelle nous a permis, non seulement de joindre quasiment tous les traducteurs et jurys, mais également d'obtenir un aperçu descriptif cohérent et lisible du fait que la composition des jurys n'avait pas considérablement changé pendant cette période. Nos conclusions restent donc subsidiaires d'un état récent du champ de la traduction et invitent à être contrastées avec une analyse historique plus détaillée sur la longue durée tenant également compte de ses transformations à long terme.

Ensuite, la dimension nationale et culturelle de l'enquête, centrée uniquement sur les prix de traduction français, restreint notre analyse à un champ de la traduction qui possède des caractéristiques particulières, lesquelles ne peuvent être appliquées à l'ensemble du paysage éditorial francophone, même si elles servent de point de départ pour une analyse comparative transnationale. A titre d'exemple, contrairement aux prix de traduction en France, le prix du Gouverneur Général du Canada relève d'une logique qui ne se laisse pas analyser à partir du clivage entre diffusion restreinte et grande diffusion. S'il n'est pas propre à la France, ce clivage prend tout de même une dimension capitale dans le contexte hexagonal. Ayant comme but de

\footnotetext{
${ }^{3}$ Nous avons suivi ici le « protocole d'études des prix » proposé par Jérôme Meizoz dans son article déjà cité « La fabrique du fétiche. Sociologie des prix littéraires ».

${ }^{4} \mathrm{La}$ division par domaines et profils récompensées et la collusion des instances donatrices (la SDGL attribue quatre prix différents) sont des facteurs qui excluent la lutte entre les prix pour le monopole du pouvoir de consécration. De là, les réponses des jurés concernant les critères de sélection des œuvres primées, ainsi que les procédures et les rituels mis en place pour la célébration des lauréats, ne diffèrent pas beaucoup entre eux.
} 
promouvoir la littérature canadienne dans l'autre langue officielle que celle dans laquelle a été écrite l'ouvrage (français ou anglais selon le cas) ${ }^{5}$ le prix du Gouverneur Général présente une forte composante politique, médiatique et financière (25000 dollars sont remis au lauréat et les éditeurs primés reçoivent une subvention de 3000 dollars pour leurs activités de promotion) qui détermine les enjeux symboliques et économiques. En témoignent la forte médiatisation du prix (les ouvrages primés reçoivent une nouvelle jaquette avec macaron et font l'objet d'une promotion très large assurée par le bureau de presse du Conseil des arts et par les éditeurs eux-mêmes), ${ }^{6}$ un appareil institutionnel solide doté d'une charte très précise stipulant les règles de sélection, d'attribution et de circulation des prix, ${ }^{7}$ une forte préséance des grands éditeurs dans la sélection et promotion des œuvres primées ${ }^{8}$ ainsi qu'une description du rôle du traducteur comme " passeur " qui révèle une volonté politique et promotionnelle de la littérature. ${ }^{9}$ On peut donc attirer l'attention du chercheur sur cette différence en signalant au passage que la volonté du prix de traduction du Gouverneur Général du Canada de promouvoir la littérature écrite en anglais et en français dans un pays bilingue exclut de la reconnaissance symbolique et économique, à la différence de la France, les traducteurs et éditeurs portant leur attention sur les langues minoritaires. D'autres enjeux, notamment de type politique et événementiel, gouvernent donc le champ de la traduction au Canada, enjeux qui ne se manifestent pas sous la même forme dans le champ de traduction français.

En outre, diverses questions liées aux modes de fonctionnement et de construction de l'autorité, ainsi qu'à la façon dont ils occupent une position dans le paysage éditorial et produisent de la valeur, se posent lorsque l'on se penche sur l'histoire des prix de traduction. Bien que notre enquête repose sur une

\footnotetext{
${ }^{5}$ Voici la réponse de Mireille Allaire, agente de relations publiques au Conseil des arts du Canada, à ce sujet: "Étant donné que le Canada est un pays avec deux langues officielles, l'anglais et le français, c'est toujours une préoccupation au Conseil des arts du Canada que la littérature canadienne-anglaise soit connue et lue par les francophones et vice versa. Le but des Prix GG en traduction est de promouvoir la littérature canadienne dans l'autre langue officielle et aussi de promouvoir l'importance du traducteur littéraire, qui joue le rôle clé d'un « passeur » dans ce contexte ». Entretien avec Mireille Allaire, réalisée par Valérie Le Plouhinec le 15 décembre 2014, inédite.

${ }^{6}$ Les auteurs, traducteurs et éditeurs des livres primés, affirme Mireille Allaire, doivent participer pleinement à toutes les activités entourant les prix, y compris la remise des prix, les réceptions, les entrevues médiatiques et les conférences de presse. Ils doivent également accepter que leur photo et des renseignements publics les concernant soient utilisés pour la promotion de ces prix et à d'autres fins promotionnelles du Conseil des arts.

${ }^{7}$ Parmi les règles de sélection et d'attribution de prix, il est important de signaler ici que les œuvres participant aux prix doivent être écrites dans une des langues officielles (anglais ou français). On voit là clairement la volonté politique du prix de traduction du GG de promouvoir démocratiquement la littérature dans un pays bilingue.

${ }^{8}$ «Seuls les éditeurs peuvent soumettre les livres; les écrivains, les traducteurs, les membres du jury, etc. ne peuvent pas proposer d'ouvrages pour les Prix GG», Entretien avec Mireille Allaire. ${ }^{9}$ En témoigne la réponse de Mireille Allaire à la question de savoir si le prix de traduction est avant tout un instrument de reconnaissance d'un traducteur par ses pairs ou un outil de promotion du rôle du traducteur littéraire dans le monde lu livre : « Bien que la reconnaissance par les pairs et la promotion du rôle du traducteur littéraire soient très importantes, avant tout, les Prix GG en traduction reconnaissent les meilleures traductions littéraires de l'année. C'est une façon de promouvoir de bons livres traduits auprès du grand public et des gens de la communauté littéraire », Entretien avec Mireille Allaire.
} 
description institutionnelle nous permettant d'interpréter les prises de position des acteurs impliqués, nous avons voulu nous concentrer uniquement dans cette première partie de la recherche sur les discours que les traducteurs eux-mêmes tiennent sur leur métier, privilégiant ainsi une approche compréhensive. Une étude descriptive sur la conformation des prix de traduction en France, sur leur histoire et leurs mécanismes de fonctionnement, ainsi que sur les rapports de force dans lesquels ils émergent et se positionnent, serait des plus intéressante. Elle permettrait, comme c'est les cas des études consacrés en France aux prix littéraires, de mieux cerner la manière dont un patrimoine culturel et un statut social circulent et s'administrent. Nous en fournissons certains éléments nécessaires, bien que lacunaires, tout au long de notre article et dans les annexes (voir notamment le tableau $\mathrm{n}^{\mathbf{0}} 2$ ), sans pour autant répondre à toutes les questions qui surgissent, et qui seront traitées dans une étude à venir.

\section{Quelle valeur de consécration pour les prix de traduction?}

L'idée directrice qui préside à la création de chaque prix, ainsi que leur rétribution économique et leurs règles de fonctionnement, peuvent nous donner d'emblée un indice pour décoder la façon dont ils se positionnent et construisent leur autorité. Conçus à la fois comme une distinction de la personne du traducteur par la récompense $d^{\prime}$ ' un travail d'exception» ${ }^{10}$ et comme une façon de " rendre visible sa figure aux yeux du public ", les prix de traduction participent à la fabrication d'un système de légitimation institutionnelle tiraillé entre l'aspiration à une excellence reconnue par un corps de spécialistes et la nécessité d'accéder à une plus grande visibilité publique. Pour garantir leur pouvoir à décréter et à imposer la valeur, ils doivent donc se doter, pour reprendre la formulation de Jérôme Meizoz à propos des prix littéraires, " de l'appareil ordinaire de l'objectivité, à savoir d'un mode d'élection, d'un mode de scrutin, de statuts fixes, etc. ». Bien que cet appareil ne soit pas toujours entièrement transparent et rigoureux, on peut distinguer certaines formes spécifiques d'organisation vouées à construire leur crédibilité.

\section{Le jugement par des pairs}

« Les jurys sont composés, sans exception, de traducteurs ». Ce système d'évaluation par les pairs constitue le pilier sur lequel repose leur autorité. « La reconnaissance de ses pairs, explique Joëlle Dufeuilly - lauréate en $2014 \mathrm{du}$ Grand prix de la traduction SGDL pour sa traduction du hongrois de Guerre et Guerre de László Kraszanahorkai -, est la plus importante des reconnaissances, et également la plus pertinente, car il s'agit d'experts qui connaissent les enjeux et les défis à relever ». Ce sentiment général est confirmé par Anne Damour, jurée du prix Maurice-Edgar Coindreau de la SGDL, pour qui le prix « est avant tout une reconnaissance du traducteur par ses pairs, car le jury est composé uniquement de traducteurs professionnels qui tiennent à leur indépendance ». Bien que ce principe ne soit remis en question ni par les jurés ni par les lauréats,

\footnotetext{
${ }^{10}$ Les citations entre guillemets correspondent aux réponses données par les lauréats et les jurés aux questionnaires. Lorsqu'elles reproduisent un sentiment général, partagé par la plupart des enquêtés, elles ne seront pas accompagnées de références particulières.
} 
il suscite des réponses nuancées lorsqu'il est formulé en relation avec la reconnaissance attribuée par le grand public. Ainsi, à la question de savoir si le prix est avant tout un instrument de reconnaissance d'un traducteur par ses pairs ou un outil de visibilité du rôle du traducteur dans le marché du livre, les réponses, assez unanimes chez les jurés et les lauréats, signalent un mécontentement général provoqué par l'absence de visibilité des prix dans le grand public. À cet égard, la réponse de Christophe Mileschi, lauréat en 2013 du Grand prix de la ville d'Arles pour sa traduction des Promesses de Marco Lodoli, est assez significative : «Idéalement, je voudrais que les prix de ce genre soient un outil de promotion du rôle du traducteur ; concrètement, je pense qu'on en est loin, et que cela reste presque uniquement un gage de reconnaissance entre pairs. C'est déjà très bien, mais on manque d'un Goncourt des traducteurs... ».

«Parce qu'ils ne fonctionnent pas comme un outil de promotion », selon la formule de Martine Rémond, lauréate en 2011 du prix Laure Bataillon de la SGDL, le volume de reconnaissance publique que les prix apportent est souvent perçu par les traducteurs comme très faible, voire comme une incapacité institutionnelle à imposer aux acteurs de la chaîne du livre (éditeurs, critiques spécialisés, etc.) et au public en général une image valorisante du traducteur. " Le public en général, ainsi que les médias, ne s'intéresse pas au rôle du traducteur. Le travail des prix de traduction à cet égard est considérable, mais il reste toujours beaucoup à faire dans ce sens-là ", lit-on en substance dans la plupart des réponses des lauréats. Il existe pourtant un travail de médiatisation des prix. En ce qui concerne les prix octroyés par la Société des gens de lettres : (Grand prix de traduction SDGL, Prix Baudelaire, Prix Gérard de Nerval, Prix Maurice-Edgar Coindreau), Cristina Campodonico, responsable communication de la SGDL, affirme : « nous envoyons des communiqués à la presse, mais vous savez bien : les prix de traduction, tout le monde s'en fiche complètement ». "Il n'y a malheureusement pas de retombées dans la presse, bien qu'il y ait des invités ! », confirme Jean-Pierre Lefèvre, juré du prix Gérard de Nerval. Même réponse pour le prix de la SFT (Société française des traducteurs) : «Des communiqués de presse sont envoyés à la presse généraliste et spécialisée. Ils sont également publiés sur le site de la SFT. L'éditeur de la traduction récompensée s'engage à en faire la promotion, notamment par un bandeau », déclare Débora Farji-Haguet, présidente du jury du prix en question. Cependant, « il est rare, disait en 2009 Florence Herbulot, ancienne présidente de la SFT, que le livre primé reçoive la bande qui donnerait, en librairie, un relief à notre prix » (107). ${ }^{11}$

\footnotetext{
${ }^{11}$ L'absence d'un bandeau ne peut pas cependant être attribuée seulement à un oubli volontaire du libraire ou de l'éditeur, « qui ne voient pas que cette stratégie fait vendre un exemplaire de plus » (Fréderic Cambourakis, éditeur du Grand Prix SGDL 2014). En effet, en interrogeant les traducteurs, les jurys et les agents chargés de la presse sur ce point, on constate que l'absence du bandeau relève aussi des problèmes de logistique. « Il faut dire que la difficulté est grande : les livres sont déjà distribués, on ne peut pas les récupérer pour poser la bande ; pour que ce soit possible, il faut ou il faudrait une réédition, mais comment l'obtenir ? », affirmait il y a encore six ans Florence Herbulot dans le même article. Ils pourraient cependant envoyer un nombre de bandeaux aux libraires, comme dans le cas de prix littéraires. On est porté à croire que s'ils ne le font pas, c'est parce que le prix reste en soi-même un argument commercial trop faible pour mériter des efforts logistiques qui exigeant un investissement important en temps et en argent.
} 
Les réponses des traducteurs et des jurés à l'égard de la visibilité de prix de traduction font penser que cette absence de visibilité médiatique est le résultat du manque d'intérêt des éditeurs et des libraires pour les traductions primées («Les éditeurs se désintéressent des prix de traduction parce qu'ils ne rapportent aucune vente »). Sans impact économique ressenti, les prix de traductions seraient ainsi exclus du système de commercialisation du livre. Or, cette exclusion, regrettée par la plupart des lauréats (« Je ne suis pas sûre que le rôle du traducteur soit vraiment promu dans le monde du livre »; "l'éditeur est souvent présent lors de la remise du prix, mais je crains qu'il ne fasse guère de promotion ni de communication ensuite... ») pourrait aussi s'expliquer par le manque d'intérêt des éditeurs pour la traduction, y compris ceux opérant dans la sphère restreinte. En effet, on peut envisager une volonté des éditeurs (consciente ou non) d'occulter le processus même de la traduction - dans la mesure où ils ne désirent pas rappeler aux lecteurs que ce qu'ils lisent est une traduction, et non le texte de l'auteur qu'ils vendent. ${ }^{12}$ En tout cas, les éditeurs ne participent pas ou peu (par la pose d'un bandeau, par la mise en scène médiatique du prix, par l'exposition du traducteur dans les évènements de commercialisation et exposition du livre, etc.) à la consécration symbolique ou économique du traducteur. Cette circonstance, qui éloigne les prix de traductions de la dynamique d'attribution de la valeur propre à la diffusion des biens culturels en grande diffusion, est vécue par les traducteurs comme une méconnaissance de leur activité. Elle permet cependant aux prix de traduction de monopoliser le pouvoir de consécration et de conserver leur légitimité. À cet égard, il est intéressant ici de les comparer avec leurs voisins littéraires. D'après Sylvie Ducas, « la mise en place [par les prix littéraires] d'une nouvelle valeur de la littérature - marchande, médiatique, spectaculaire, démocratique et consensuelle » a bousculé " les rapports entre jugement critique et valeur littéraire tels qu'on pouvait les penser dans les sphères lettrées traditionnelles » (n.p.). Cette dynamique est pourtant absente des prix de traduction. À l'abri des contraintes économiques imposées par le « dispositif médiatico-publicitaire placé au cœur des stratégies éditoriales et de l'industrie du livre » (n.p.), pour reprendre la formulation de Sylvie Ducas par rapport aux prix littéraires, et n'étant octroyés que par un corps relativement indépendant, ils ne favorisent pas le mélange entre instances de diffusion et instances de consécration, ce qui leur permet de garder leur autonomie et de se pencher sur la sphère de production restreinte. C'est précisément ce que met en valeur la responsable

Toutefois, la situation évolue peu à peu: depuis 2012, la SFT fait poser un bandeau sur les livres couronnés par le prix Pierre-Françoise Caillé, et à l'occasion de la remise du Grand prix de la ville d'Arles, lors des Assises de la traduction, on trouve des livres avec bandeau dans les deux grandes librairies d'Arles. Cela s'explique par l'importance symbolique du prix pour la ville et par l'engagement de ces deux libraires dans l'activité culturelle de la ville, ainsi que par la relative simplicité de mise en place du dispositif.

${ }^{12}$ Cette hypothèse doit encore être confirmée par un questionnaire aux éditeurs eux-mêmes. Il existe cependant, comme l'a signalé Lawrence Venuti pour le cas anglophone dans son ouvrage intitulé L'invisibilité du traducteur (The translator's Invisibility. London/New York: Routledge, 1995), l'idée très répandue que la traduction doit se lire comme s'il s'agissait d'un original, autrement dit sans faire apparaître la figure du traducteur-médiateur. 
communication de la SGDL, Cristina Campodonico, dans ses réponses au questionnaire envoyé :

La SGDL se différencie des autres grands prix médiatisés par une volonté de découverte et de mise en valeur d'auteurs et de traducteurs talentueux et méconnus, loin du cirque commercial des prix médiatisés de la rentrée littéraire - qui, il est vrai, couronnent un peu toujours les mêmes éditeurs poids lourd et les mêmes auteurs déjà populaires et connus. La forte présence de petites maisons d'édition indépendantes dans le palmarès est une volonté des jurés : c'est tout à fait volontairement qu'ils ne souhaitent pas privilégier les grosses machines éditoriales qui ont déjà une forte visibilité. Les jurés lisent beaucoup et vont volontiers vers des livres moins médiatisés, des petites maisons innovantes, indépendantes. De même, la difficulté d'un livre ne leur fait pas peur, ou plutôt le potentiel commercial de l'œuvre primée n'entre pas en ligne de compte. Ce qui les intéresse est uniquement le talent du lauréat, ils n'ont pas d'autre critère de jugement.

En effet, si l'on regarde le tableau des œuvres primées, on peut y constater une forte présence des petites maisons d'édition (Arléa, Zulma, Gallmeister, Philippe Picquier, Tristam, Quidam, Le Bruit du Temps, Cambourakis, Zoé, etc.) au détriment des grandes machines éditoriales (voir le tableau $n^{\circ} 1$ ). Pour un total de quatre-vingts œuvres couronnées pendant les quatre dernières années (comprenant les huit prix de traductions retenus pour notre enquête) Gallimard, Actes Sud et Robert Laffont n'apparaissent que deux fois, suivis par Le Seuil, Fayard et Albin Michel, qui n'apparaissent qu'une seule fois respectivement.

\section{La sélection des ouvrages}

Cette volonté d'indépendance vis-à-vis des contraintes économiques de la sphère de grande production, dont témoigne la prédilection de petites maisons d'édition pour les traductions publiées, est assurée aussi par les critères de sélection des ouvrages. Tout d'abord, à la différence des prix littéraires, où les éditeurs jouent un rôle fondamental en proposant des ouvrages aux jurys des prix, ce sont les jurés eux-mêmes qui proposent les ouvrages concourant pour le prix: « Nous établissons une liste nous-mêmes, les éditeurs n'envoient que les titres que nous leur demandons », affirme Jean-Pierre Lefèvre. Ce critère est commun à quasiment tous les prix (avec l'exception du prix Pierre-François Caillé attribué à un traducteur en début de carrière). Ce faisant, les jurés des prix de traduction cherchent à privilégier des critères de sélection autonomes, basés non pas sur le prestige et la capacité de production et diffusion de certaines maisons d'édition, mais sur la qualité des ouvrages traduits. ${ }^{13}$

Cette propension à l'autonomie se voit confirmée par le refus de prendre en considération le profil du traducteur. Avec l'exception du Grand prix de la traduction de la SGDL, qui récompense l'ensemble de la carrière d'un traducteur, et du prix Pierre-François Caillé, qui encourage le début de carrière d'un traducteur, les jurés affirment ne pas prendre en compte la réputation du

\footnotetext{
13 On peut se demander si cette insistance sur une présélection autonome par rapport au jugement des maisons d'édition n'est pas aussi une manière de faire de nécessité vertu, car elle peut s'expliquer par le manque d'intérêt des éditeurs pour les prix de traduction, qui n'envoient pas toujours spontanément les livres aux jurys.
} 
traducteur: « Nous ne tenons pas compte de la notoriété du livre ou du traducteur, non plus que celle de l'éditeur ", affirme Jean-Pierre Lefèvre, juré du prix Gérard de Nerval. « Il n'y a que le résultat, la production écrite, et, donc, la qualité du travail de traduction, qui compte pour les jurés, confirme Cristina Campodonico, au point que quand un éditeur leur propose d'envoyer un dossier de présentation, une biographie du traducteur, etc., ils n'en veulent même pas parce qu'ils ne jugent que le texte, sans autre influence $»{ }^{14}$ La notoriété du traducteur est ici perçue par les jurys comme un critère hétéronome, le plus souvent lié à la culture du spectacle, à la capacité de l'acteur à accumuler un capital de visibilité, qui pourrait détourner leur attention de ce qui compte réellement pour eux : la qualité du travail de traduction, seule valeur qui mérite d'être récompensée. Pour expliquer ce refus de considérer le parcours du traducteur, on peut aussi évoquer la réticence d'une grande partie des traducteurs littéraires vis-à-vis des formes de professionnalisation, y compris les diplômes. On pourrait supposer qu'il existe, à l'intérieur même des jurys, un antagonisme entre traducteurs à plein temps et traducteurs universitaires (avec des degrés d'investissement, des parcours et des niveaux de formation différents) qui pourrait provoquer des conflits d'intérêts. ${ }^{15}$ Mais la proximité des traducteurs littéraires, par leur objet et par leurs conditions de travail, avec le régime de production artistique moderne, permet à certaines valeurs, comme l'originalité et la singularité, de prendre le devant de la scène, aussi bien chez les traducteurs occasionnels que chez les traducteurs à plein temps, au détriment des valeurs collectives comme la condition d'accès à la profession (Kalinowski). Pourtant, nous verrons, lors de l'analyse du profil des traducteurs récompensés, qu'ils ont tous en commun un fort capital culturel. Cette circonstance s'explique non pas par la volonté calculée des jurés à primer les trajectoires exceptionnelles, sanctionnées par des institutions scolaires ou même par le succès public, mais par la préférence pour les ouvrages « rares » et réputés difficiles, accessibles uniquement à un public restreint.

Le travail de sélection et d'évaluation est donc assumé et revendiqué par les jurés comme une activité indépendante des contraintes hétéronomes imposées par la machine industrielle, voire comme une vocation : " Il s'agit pour le juré, affirme Claire de Oliveira, d'un travail considérable et non rémunéré, que nous faisons pour promouvoir une traduction de qualité supérieure ». Pour sa part, Florence Herbulot décrivait le corps de spécialistes qui compose le jury du prix Pierre-François Caillé de la STF comme des « gens dévoués à la tâche, qu'ils assument avec courage et assiduité, mettant beaucoup de sérieux dans le jugement de la qualité des livres qu'ils ont à lire chaque année » (Herbulot 105). Vécu comme une vocation détachée des pressions externes et des exigences professionnelles, le système d'évaluation par les pairs est donc pour les jurés un garant de leur légitimité, fondée uniquement sur les critères d'excellence et sur l'expertise des agents de consécration. Les conditions de

\footnotetext{
${ }^{14}$ Seul le prix Pierre-François Caillé prend en compte le curriculum vitae du traducteur. Cette exception s'explique cependant autrement : il s'agit en effet d'un prix découverte qui récompense le travail d'un jeune traducteur, raison pour laquelle la trajectoire du traducteur est prise en compte

${ }^{15}$ Les traducteurs occasionnels revendiquant la valeur symbolique de la traduction, renonçant ainsi à la personne juridique du traducteur au nom du personnage, et les traducteurs à plein temps revendiquant la valeur économique du métier et la personne juridique du traducteur.
} 
travail du traducteur littéraire ${ }^{16}$ et son rapprochement du régime de création artistique, favorisent cette tendance à définir et à valoriser ce métier à partir du système vocationnel. Ce faisant, le traducteur renonce à la seule revendication matérielle de sa profession. ${ }^{17}$ Cela explique aussi que les prix de traduction (dont la faible dotation économique ne permet ni indépendance vis-à-vis du marché ni amélioration des conditions de travail du traducteur) se définissent eux aussi de manière vocationnelle en insistant uniquement sur les aspects symboliques.

Or, cette valeur symbolique accordée par les prix ne semble pas se traduire par une amélioration du statut des lauréats (liberté de choix des auteurs, plus grande autorité pour proposer des auteurs aux éditeurs, nombre plus important de contrats). Ces derniers sont unanimes: les prix ont un impact limité sur leur carrière socioprofessionnelle. « Ni plus grande liberté, ni plus grande autorité, ni plus de contrats », affirme sur ce point Martine Rémond. Dans le meilleur des cas, nous raconte Joëlle Dufeuilly, pourtant couronnée en 2014 par le Grand prix SGDL pour l'ensemble de son œuvre, l'un des plus prestigieux au sein de la profession, le prix peut déclencher une intervention plus active dans la sphère du livre « (invitations à des tables rondes sur la traduction, coopération avec une agence littéraire, demande d'expertise de traductions, etc.), mais pas plus de choix pour les auteurs à traduire ». Ainsi, si les prix peuvent dans certains cas apporter au traducteur une plus grande estime aux yeux des éditeurs (" disons que j'ai gagné en estime pour l'éditrice, qui avait déjà une bonne opinion de moi, mais cela ne m'a pas ouvert de portes supplémentaires », affirme Christine Raguet, lauréate du prix Baudelaire en 2012), ils ne sont pas synonymes d'une plus grande liberté éditoriale (pour proposer des auteurs aux éditeurs) ni d'une plus grande indépendance vis-à-vis des contraintes économiques. Ce dernier point s'explique en grande partie, on l'a vu, par la faible rémunération des prix. Pour la plupart, elle oscille entre deux mille et cinq mille euros. ${ }^{18}$ De toute évidence, cette récompense économique ne permet pas au traducteur vivant de la traduction de se libérer des contraintes du marché. Il faut toutefois nuancer ce constat. En effet, la plus grande partie de lauréats interviewés est constituée de traducteurs ayant capitalisé dans un champ discursif spécifique ou un type de littérature en particulier. Qu'ils soient universitaires travaillant à temps partiel pour la traduction, donc bénéficiant d'une indépendance économique liée à leur statut, ou traducteurs à plein temps

\footnotetext{
${ }^{16}$ Nous pensons à l'isolement et l'individualisation du rapport au travail, la précarité juridique et économique, la faible professionnalisation ainsi qu'à la reconnaissance d'un produit et non pas d'un statut

${ }^{17}$ Cet aspect est suggéré par Isabelle Kalinowski dans son article déjà cité. Selon Kalinowski, le fait qu' " il n'existe pas, en France, de syndicat des traducteurs littéraires, mais une Association des traducteurs littéraires (ATLF), créée en 1973 et distincte du syndicat des traducteurs "techniques", la Société française des traducteurs (SFT), fondée en 1947 », montre « la réticence des traducteurs littéraires à l'égard des formes de professionnalisation les plus structurées » et empêche que ses revendications, le plus souvent énoncées comme " codes de bons usages », soient prises au sérieux par les acteurs de la chaine du livre, car elles ne possèdent pas une valeur légale. (« La vocation au travail de traduction », 49-50)

$182000 €$ (Prix Maurice-Edgar Coindreau, Pierre-François Caillé, Prix Baudelaire) ; $4000 €$ (Prix Gérard de Nerval) ; $5000 €$ (Grand prix de traduction littéraire de la ville d'Arles et Prix Laure-Bataillon, doté d'une récompense de $10000 €$ attribuée conjointement à l'écrivain étranger et à son traducteur en langue française) ; $6000 €$ (Grand prix de la SGDL).
} 
jouissant d'une forte notoriété, ayant donc développé des compétences spécifiques par lesquelles ils sont reconnus et sollicités dans le marché de l'édition, les traducteurs lauréats disposent d'un certain degré d'autonomie qui leur permet de choisir parmi les commandes proposées par les éditeurs. Cette particularité explique la tendance chez les traducteurs interviewés à définir leur activité, même pour ceux qui vivent de la traduction, comme une vocation, tout en insistant sur la valeur symbolique du prix et non sur sa valeur économique. ${ }^{19}$

Pour revenir à notre point de départ, l'ambivalence entre la légitimité conférée par le jugement des pairs et la nécessité d'accéder à une visibilité plus large est ressentie et valorisée par les lauréats de deux manières : d'un côté, l'évaluation et la reconnaissance par les pairs fonctionnent pour eux comme un indice de la légitimité du prix, échappant ainsi aux soupçons de connivence entre l'agent consacrant et les pressions externes, souvent liés aux intérêts purement commerciaux. De l'autre, elles fonctionnent comme une compensation d'un déficit d'image attribuable à la méconnaissance publique d'un métier le plus souvent vécu comme n'étant pas assez reconnu. On peut citer à cet égard les témoignages de quelques traducteurs interviewés :

Le public peut être abusé quant à la pertinence de ce qu'il lit. Les pairs valident en quelque sorte un succès qui peut être porté par les médias sans examen de la pertinence de la traduction. Il donne aussi confiance, pour cette même raison, au traducteur luimême, toujours en proie au doute... (Jean-François Lefèvre, juré du prix Gérard de Nerval.)

Il m'arrive assez souvent de rencontrer des gens, dont des amateurs de littérature, qui n'ont qu'une vague notion de ce en quoi consiste le métier de traducteur (c'est compréhensible : on n'en parle jamais !) : pour eux, il suffit de maitriser une langue pour savoir la retranscrire. Pour un peu, on me dirait que la traduction consiste à faire du mot à mot! Je ne m'en offusque pas, c'est le genre de choses dont on ne prend conscience qu'en y étant confronté, mais je me rends compte que peu de personnes savent que deux langues ne diffèrent pas seulement en vocabulaire, mais aussi en construction, en façon de "penser" le langage, en environnement culturel - sans mentionner l'écriture, la "patte" d'un auteur, qui parfois triture le langage à sa manière toute personnelle. Les traducteurs et, je pense, la plupart des éditeurs, eux, savent ce que c'est, puisqu'ils font face à ces problèmes dans leur travail. Ils savent que le choix d'un bon traducteur est essentiel dans le fait de faire passer un livre d'une langue à une autre, d'un environnement à un autre. (Jean-Christophe Salaün, lauréat en 2014 du Prix Pierre-François Caillé pour sa traduction de l'islandais du roman La Femme à 1000 degrés de Hallgrimur Helgason).

Je pense qu'il y a différents types de publics :

- ceux qui n'ont pas du tout idée de ce que peut être la traduction. On parle une langue (plus ou moins vaguement) donc on peut traduire. Et puis, ils ne voient pas le temps que cela peut prendre, les recherches, l'implication ;

- ceux qui connaissent un peu le monde des lettres et qui perçoivent bien que chaque texte est un défi d'un autre ordre et qu'un traducteur joue justement un rôle essentiel au niveau de la réception d'un texte ;

- les pairs : en général, il existe du respect entre traducteurs littéraires. J'ai

l'impression que tous les gens que je côtoie font leur travail très sérieusement et sont passionnés. (Christine Raguet, lauréate en 2012 du Prix Baudelaire pour 
sa traduction de Soucougnant et Éclairs de chaleur de David Chariandy et Olive Senior.)

Reposant uniquement sur la reconnaissance de la valeur de l'auteur, le succès éditorial d'une œuvre traduite est donc vécu comme une occultation du travail du traducteur. Dans ces termes, les demandes de reconnaissance publique des traducteurs ne contredisent pas véritablement la légitimité conférée par le jugement des pairs, mais s'avèrent plutôt déterminantes pour l'accréditation d'une fonction longuement tenue dans l'ombre. C'est ainsi que Christophe Mileschi le décrit, exprimant un sentiment assez généralisé chez les traducteurs :

Il me semble que le public, très souvent, ne "perçoit" tout simplement pas le traducteur. Du reste, même dans les articles "spécialisés", il n'est pas rare qu'on trouve un éloge de la langue, de l'écriture, du style d'un écrivain, sans aucune mention du fait qu'il a été traduit par quelqu'un... exactement comme si l'auteur de l'article commentait l'œuvre originale, alors qu'il y a eu accès dans la traduction française. Ça frise parfois le ridicule. Un peu comme si, à chaque fois qu'un musicien soliste donnait un concert, les critiques ne parlaient que de la partition, sans jamais faire allusion à l'interprétation... Quant aux traducteurs, ils ont évidemment conscience de leur propre rôle et du rôle de leurs consœurs et confrères (nous qui traduisons pouvons difficilement oublier que, si un livre écrit dans une autre langue est lisible en français, c'est qu'un traducteur se cache quelque part !). Et je pense que, souvent, ils souffrent de la méconnaissance dont leur travail fait l'objet.

L'absence de médiatisation des prix de traduction, leur relatif isolement du grand public et le peu d'intérêt qu'ils suscitent chez les éditeurs (pour qui ces prix ne constituent pas un argument commercial) deviennent un adjuvant de leur autonomie. Grands absents des circuits de commercialisation, les prix de traduction ne se voient pas confrontés, comme leurs voisins littéraires, à la nécessité de construire un discours légitimant, voire de disculpation, de leurs pratiques. La défiance vis-à-vis du succès commercial, qui pèse toujours aujourd'hui sur les gagnants à court terme de la partie littéraire, a fait des prix littéraires, de plus en plus soupçonnés « d'une aliénation suspecte à des intérêts éditoriaux ayant peu à voir avec la consécration littéraire» (Ducas n.p), un objet privilégié des critiques des auteurs et des académiciens. Cela les amène, comme l'a démontré Sylvie Ducas, à une lutte acharnée pour le pouvoir de consécration dans laquelle émergent de nouveaux discours légitimants fondés non pas sur une politique culturelle propre à l'élite intellectuelle, perçue par les instances médiatiques comme élitiste et antidémocratique, mais sur des valeurs communautaires liées « à la démocratisation des pratiques culturelles » (Grand Prix des lectrices de Elle, prix du Livre Inter ou de RTL/Lire) (Ducas n.p.). Moins exposés aux contraintes de la médiatisation et aux réalités marchandes inhérentes à l'industrie culturelle, les prix de traduction ne semblent pas être affectés par cette dynamique. Mais si leur statut en tant qu'entité consacrante n'est pas mis en question par les lauréats, en vertu du principe qui affirme que la spectacularisation est toujours suspecte et le jugement des pairs toujours légitime, les réponses de ceux-ci, nous l'avons vu, laissent deviner un mécontentement vis-à-vis de l'absence de visibilité de leurs prix dans le grand public.

Les demandes de reconnaissance publique du traducteur se trouvent donc tiraillées entre une définition professionnelle du métier qui revendique la personne juridique du traducteur et une définition vocationnelle qui revendique 
«l'idéologie charismatique de la création» (Bourdieu 279). Elles ne doivent pas pourtant être interprétées comme une mise en question du principe de jugement par des pairs sur lequel reposent les profits symboliques (prestige et légitimité) de leur activité. Sans renoncer complètement aux réalités exigibles de sa profession (notamment en ce qui concerne les rapports de force avec les éditeurs et une éventuelle protection de la part des institutions gouvernementales pour garantir leur autonomie), la définition vocationnelle de son métier devient pour le traducteur littéraire une sorte de consolation, gratifiante symboliquement mais décevante économiquement, de la précarité juridique et matérielle de son métier. $^{20}$

\section{Qu'est-ce qu'un bon traducteur ? Les traducteurs récompensés}

Les critères d'attribution d'un prix, le profil des lauréats, le profil des traductions primées, la tendance en matière de langues sources et de maisons d'édition privilégiées par les jurés, ainsi que les procédés discursifs cherchant à produire un effet de "notoriété », constituent autant d'indices nous permettant d'éclairer la façon dont les prix de traduction fabriquent une image régulatrice du traducteur et de son métier. Bien qu'elle ne soit pas universellement admise, et qu'il existe au sein même de chaque prix des prises de position diverses en fonction de l'histoire et de la position occupée par chacun des agents impliqués, on peut constater, dans les réponses des jurés interviewés, une tendance à privilégier et mobiliser les dispositifs de légitimation et de valorisation propres au champ restreint.

\section{Un traducteur « hors du commun » : rareté et singularité du produit}

Souvent décrite par les jurés comme un travail « hors du commun » (« traduction de qualité supérieure », " traduction exceptionnelle », « meilleure traduction du meilleur livre »), la traduction primée, ainsi que son traducteur, acquièrent par ce fait le droit d'entrée dans le palmarès du champ de la traduction. Cet espace restreint, d'autant plus charismatique qu'il ne définit pas explicitement ses critères de distinction, est investi de certaines valeurs qui se trouvent le plus souvent associées aux cadres de perception d'une élite culturelle pour laquelle la valeur esthétique n'est pas réductible à la valeur commerciale. Ainsi, la rareté et la singularité du produit opèrent comme les critères prépondérants sur lesquels est fondée l'évaluation. Jouant un rôle fondamental à l'heure de la sélection et du couronnement d'une traduction, la complexité de l'ouvrage à traduire devient un critère incontournable pour la définition de ce qu'est un bon traducteur.

On peut citer à cet égard la traduction par Nicolas Richard d'Enig Marcheur, prix de traduction Maurice-Edgar Coindreau 2013. L'ouvrage, réputé intraduisible, avait attendu trente ans avant d'être traduit. L'auteur luimême, Russell Hoban, n'était pas favorable à une traduction, qu'il jugeait infaisable. Plusieurs « stars » de la traduction (Claro, Bernard Hoepffner)

\footnotetext{
20 « La France - au niveau ministériel - ne soutient pas assez les traducteurs d'exception. Or certains d'entre eux ne vivent que de leur activité de traduction, et tous ont besoin d'être reconnus par les institutions et le grand public », affirme Claire de Oliveira, juré du prix Gérard de Nerval et lauréate du même prix en 2008 pour sa traduction d'Avidité de Elfriede Jelinek).
} 
avaient été sollicitées avant Richard, mais ont reculé devant l'immensité de la tâche. La langue de départ (le riddleyspeak) se caractérise, selon la formule de Marc Chénetier, juré du prix, par un anglais «concassé, rabouté, soumis à la fission » (Chénetier n.p.), saturé de télescopages phonétiques et difficilement compréhensible à l'écrit. Le traducteur a donc dû recréer un équivalent en français (le parlénigm) pour restituer la singularité du texte original. C'est justement ce travail de recomposition du texte, voire d'invention d'une langue, qui lui a valu le prix (« une langue peu commune a trouvé son équivalent et vit là, sous nos yeux ébahis » (n.p.) affirmait Marc Chénetier lors de la remise du prix). Le travail acharné sur la forme, la probité intellectuelle, la difficulté de la traduction, deviennent donc pour le jury les critères qui caractérisent un bon traducteur. Richard, qualifié par la presse de " traducteur de l'extrême » (Fargeon n.p.), définit quant à lui son travail de traduction comme une création : «Quand on m'a proposé le livre, je n'étais pas le premier à m'y essayer, mais ce qui a emporté ma décision de le traduire, c'est que j'ai vu ce texte comme un grand poème » (Bouchard 6-7).

\section{La « sensibilité artistique du traducteur»}

Un autre exemple de cette revendication de la traduction comme un travail acharné sur la forme, qui rapproche le traducteur de l'artiste, et notamment de l'artiste pur, est la référence à la sensibilité artistique que doit posséder le traducteur pour rendre dans la langue cible la « musicalité », les « tons », les « couleurs » de l'œuvre originale (« Le traducteur, le bon traducteur en tout cas, doit avoir une sensibilité artistique qui n'est pas donnée à tout le monde »). En 2012, à l'occasion de la remise du prix Maurice-Edgar Coindreau à Pierre Demarty pour sa traduction de l'anglais des Foudroyés de Paul Harding, Marc Chénetier décrit le traducteur comme « un artiste du verbe », car d'après lui, en lisant sa traduction, on s'aperçoit dès les premières pages qu'il s'agit « d'une traduction attentive, amoureuse et dévouée, dont le chromatisme moelleux et les arêtes vives semblent naturellement s'imposer, fidèles aux reliefs et au grain d'un original où dominent les jeux de l'ombre et de la lumière, la fascination du détail, le goût artisanal de la nuance, du juste cliquet et de la brosse douce » (Chénetier «Démarty»: n.p.). L'oreille musicale du traducteur, au-delà de la maîtrise de la langue, est aussi mise en évidence par les jurés. C'est le cas de Sika Fakambi, lauréate du prix Laure-Bataillon et du prix Baudelaire en 2014 pour sa traduction de l'anglais (Ghana) de Notre quelque part de Nii Ayikwey Parkes. Le récit, qui se déroule au Ghana, fait intervenir à la fois des personnages urbains et des villageois, possédant chacun un registre où se mélangent tournures grammaticales locales, onomatopées, mots issus des langues vernaculaires - parfois laissés tels quels, avec un glossaire disponible en ligne - et langage du colonisateur. Pour rendre cette langue angloghanéenne, Sika Fakambi a dû faire un travail d'adaptation, inspiré du langage de l'Afrique de l'Ouest francophone, qui exige « beaucoup d'inventivité et de créativité », mais aussi « une très grande finesse pour ne pas trahir la musique et la saveur très particulières de cette langue ". Citons encore le cas de la traduction de Christophe Salaün, lauréat du prix Pierre-François Caillé 2014 pour sa traduction de La Femme à 1000 degrés de Hallgrimur Helgason. Le texte source, fortement marqué par l'oralité, les jeux de mots et les inventions 
langagières en islandais, se caractérisait par un langage " très rythmé » avec nombreuses allitérations et assonances. Le jury, malgré sa méconnaissance de l'islandais, affirme avoir été impressionné par l'« oreille » du traducteur, qui a su produire, avec une virtuosité évidente, un texte empreint d'un « rythme » et d'« une musicalité extrêmement maîtrisés ».

\section{Le traducteur à fort capital culturel}

La complexité de l'œuvre pour laquelle il est récompensé rehausse aussi le capital culturel du traducteur. Bien que la trajectoire des traducteurs ne soit pas prise en compte par les jurés à l'heure de leur sélection et évaluation, à l'exception du prix découverte Pierre-François Caillé, les résultats montrent qu'il existe dans tous les prix une disposition à récompenser des traducteurs dotés d'un fort capital culturel, seuls capables de traduire des ouvrages réputés « complexes et difficiles ». En témoigne leur niveau d'étude plus que supérieur (la plupart, détenteurs de diplômes de deuxième et troisième cycle), la maîtrise de plusieurs langues et leur appartenance aux fractions intellectuellement dominantes (professeurs universitaires, écrivains, éditeurs). Ce fait est assez significatif si l'on considère que le traducteur n'ayant pas capitalisé dans un domaine particulier, en l'occurrence la traduction littéraire des ouvrages appartenant par ces caractéristiques formelles à la sphère de production restreinte, considérées a priori comme les seules susceptibles de conférer une notoriété au traducteur, se voit automatiquement exclu du système de consécration et, en conséquence, de l'image symboliquement dominante du traducteur.

Cette prédisposition à primer les traducteurs littéraires dotés d'un fort capital culturel, donc aptes à traduire de textes difficiles, se voit aussi confirmée par la tendance des jurys à privilégier les « langues rares ». À l'exception des prix Baudelaire, Maurice-Edgar Coindreau et Gérard de Nerval, dont l'objectif est à la fois de mettre en valeur la figure du traducteur et de promouvoir la littérature de langue anglaise et allemande respectivement, ${ }^{21}$ les traductions primées démontrent une forte présence de langues dominées dans la cartographie linguistique mondiale (malayalam, chinois, hongrois, hébreu, catalan, islandais, néerlandais, grec et russe). La difficulté de la langue devient donc un signe de distinction et place le traducteur dans la sphère de production restreinte, confirmant ainsi l'opposition volontaire entre la valeur commerciale et la valeur symbolique qu'entretiennent les prix de traduction. En témoigne aussi, comme nous l'avons déjà signalé plus haut, la propension des jurys à couronner les traductions publiées par les maisons indépendantes, excluant ainsi du palmarès de la traduction, et de la définition symboliquement dominante du traducteur, les traducteurs appartenant à la sphère de la grande production.

\section{En manière de conclusion}

\footnotetext{
${ }^{21}$ Dans ce cas, le traducteur est aussi décrit le plus souvent comme un «passeur », comme un « introducteur », comme un « découvreur », etc. Voici les propos de Anne Damour, juré du prix Maurice-Edgar Coindreau, à ce propos : « Ce prix a été créé en 1981 à l'initiative de Michel Gresset pour rendre hommage à Maurice-Edgar Coindreau, passeur de littérature américaine, traducteur entre autres de Faulkner, Hemingway, Steinbeck... il s'agissait pour nous de continuer après lui à faire mieux connaître et à promulguer la littérature américaine ».
} 
Ainsi, seul le traducteur littéraire, notamment le traducteur des ouvrages réputés difficiles, peut obtenir, en son nom, une notoriété sur le marché de la traduction; et engager, à partir de là, son image publique dans les demandes de reconnaissance institutionnelle. On peut donc conclure que la figure du traducteur susceptible d'être reconnue et consacrée par un prix ne peut se révéler que si le texte traduit réunit deux conditions. Tout d'abord, il doit être doté d'une "fonction-auteur", au sens d'une création individuelle repérable formellement et historiquement, ce qui permet au traducteur de revendiquer à son tour une autorité (au double sens du mot) dans le processus de recréation du texte. Ensuite, le texte traduit doit s'insérer dans un circuit de diffusion et de commercialisation restreint, ce qui permettra au traducteur de réclamer une notoriété sanctionnée par les instances de consécration, instances qui privilégient, par les dispositions propres aux agents consacrants, des « ouvrages hors du commun » ou réputés " difficiles », tout en proscrivant ainsi la logique de rentabilité et de commercialisation propre à la sphère de grande production. En effet, en regardant le parcours des traducteurs récompensés, ainsi que les langues, les traductions et les maisons d'édition primées, on constate que les prix de traduction établissent une hiérarchie entre les producteurs, les modes de consommation et les canaux de diffusion qui reproduit la division entre la sphère restreinte et la sphère de grande diffusion propre aux biens symboliques. ${ }^{22} \mathrm{En}$ ce faisant, ils mobilisent les cadres de perception de la sphère symboliquement dominante, produisant ainsi une image régulatrice du « bon traducteur ». Bien qu'elle ne soit pas explicitement définie, elle participe à certaines valeurs (rareté, vocation, originalité, désintéressement) qui renvoient à ce que Nathalie Heinich a appelé le « régime de singularité » (Ce que l'art).

\footnotetext{
${ }^{22}$ Voir "L'économie des biens symboliques ", Cours du Collège de France à la Faculté d'Anthropologie et de Sociologie de l'Université Lumière Lyon, Cahiers du groupe de Recherche sur la Socialisation 13 (2e trim.1994). Lyon: Université Lumière Lyon 2.
} 


\section{Bibliographie}

Allaire, Mireille. Entretien personnel avec Valérie Le Plouhinec. 15 décembre 2014.

Bourdieu, Pierre. Les règles de l'art. Paris: Seuil, 1992.

Chénetier, Marc. « Nicolas Richard. » Société des gens de lettres. SDGL.org. . Juin 2013.

--. « Pierre Demarty. » Société des gens de lettres. SDGL.org.

Ducas, Sylvie. «Prix littéraires en France : consécration ou désacralisation de l'auteur? » COnTEXTES 7 (mars 2010).

Heilbron, Johan et Gisèle Sapiro. « La traduction littéraire, un objet sociologique. " Actes de la recherche en sciences sociales 144 (sep. 2002) : 3-5.

Heinich, Nathalie. Ce que l'art fait à la sociologie. Paris: Éditions de Minuit, 1998.

---. « Les traducteurs littéraires : 1'art et la profession. » Revue française de sociologie 25.2 (1984): 264-80.

Herbulot, Florence « Le Prix Pierre-François Caillé de la Traduction. » Traduire 221 (2009): 104-11.

Kalinowski, Isabelle. « La vocation au travail de traduction. » Actes de la recherche en sciences sociales 144 (2002) : 47-54.

Richard, Nicolas. « Le Grand Boum de Nicolas Richard. » Entretien avec Valérie Le Plouhinec. Translittérature 45 (été 2013): 6-14.

---. « Traduire Pynchon, un sacerdoce enthousiasmant. » Entretien avec Henri-

Pierre Fargeon. Télérama. 12 novembre 2013.

\section{Annexes jointes}

- Annexe $\mathrm{n}^{\circ} 1$ : Lauréats 2011-2014

- Annexe $\mathrm{n}^{\circ} 2$ : Synthèse des prix étudiés 